\title{
Targeting ferroptosis for cancer therapy: exploring novel strategies from its mechanisms and role in cancers
}

\author{
Minlin Jiang ${ }^{1,2} \wedge$, Meng Qiao ${ }^{1,2}$, Chuanliang Zhao ${ }^{3}$, Juan Deng ${ }^{1,2}$, Xuefei Li $^{1}$, Caicun Zhou ${ }^{1}$ \\ ${ }^{1}$ Department of Medical Oncology, Shanghai Pulmonary Hospital, Tongji University Medical School Cancer Institute, Tongji University School \\ of Medicine, Shanghai, China; ${ }^{2}$ Tongji University, Shanghai, China; ${ }^{3}$ Department of Otolaryngology, Tenth People's Hospital of Tongji University, \\ Shanghai, China \\ Contributions: (I) Conception and design: M Jiang, X Li, C Zhou; (II) Administrative support: X Li, C Zhou; (III) Provision of study materials \\ or patients: M Jiang, M Qiao, C Zhao, J Deng; (IV) Collection and assembly of data: M Jiang, M Qiao, C Zhao, J Deng; (V) Data analysis and \\ interpretation: M Jiang, M Qiao, C Zhao, J Deng; (VI) Manuscript writing: All authors; (VII) Final approval of manuscript: All authors. \\ Correspondence to: Caicun Zhou. Department of Medical Oncology, Shanghai Pulmonary Hospital, Tongji University Medical School Cancer \\ Institute, Tongji University School of Medicine, No 507 Zhengmin Road, Shanghai 200433, China. Email: caicunzhoudr@163.com; Xuefei Li. \\ Department of Medical Oncology, Shanghai Pulmonary Hospital, Tongji University Medical School Cancer Institute, Tongji University School of \\ Medicine, No 507 Zhengmin Road, Shanghai 200433, China. Email: bug_lily2003@163.com.
}

\begin{abstract}
Ferroptosis is a novel form of non-apoptotic regulated cell death (RCD), with distinct characteristics and functions in physical conditions and multiple diseases such as cancers. Unlike apoptosis and autophagy, this new RCD is an iron-dependent cell death with features of lethal accumulation of reactive oxygen species (ROS) and over production of lipid peroxidation. Excessive iron from aberrant iron metabolisms or the maladjustment of the two main redox systems thiols and lipid peroxidation role as the major causes of ROS generation, and the redox-acrive ferrous (intracellular labile iron) is a crucial factor for the lipid peroxidation. Regulation of ferroptosis also involves different pathways such as mevalonate pathway, P53 pathway and p62-Keap1-Nuclear factor (erythroid-derived 2)-like 2 (Nrf2) pathway. Ferroptosis roles as a double-edged sword either suppressing or promoting tumor progression with the release of multiple signaling molecules in the tumor microenvironment. Emerging evidence suggests ferroptosis as a potential target for cancer therapy and ferroptosis inducers including small molecules and nanomaterials have been developed. The application of ferroptosis inducers also relates to overcoming drug resistance and preventing tumor metastasis, and may become a promising strategy combined with other anticancer therapies. Here, we summarize the ferroptosis characters from its underlying basis and role in cancer, followed by its possible applications in cancer therapies and challenges maintained.
\end{abstract}

Keywords: Cancer; ferroptosis; immune; tumor microenvironment

Submitted Feb 26, 2020. Accepted for publication Jul 06, 2020.

doi: $10.21037 /$ tlcr-20-341

View this article at: http://dx.doi.org/10.21037/tlcr-20-341

\section{Introduction}

In multicellular organisms, cell death is an indispensable homeostatic mechanism to maintain tissue morphology and function (1). Cells may die from a biologically uncontrolled process called accidental cell death (ACD), or regulated cell death (RCD) that involves closely coordinated signal cascades with tight structure and dedicated molecular mechanisms (2). The form of cell death included three categories historically: apoptosis, autophagy, and necrosis (3). Apoptosis is the traditionally well-known RCD. However,

\footnotetext{
$\wedge$ ORCID: 0000-0001-9812-7912.
} 
Table 1 Cell morphology, biochemical features, and key regulators of ferroptosis, apoptosis, necroptosis

\begin{tabular}{|c|c|c|c|}
\hline Type of cell death & Cell morphology & Biochemical features & Key regulators \\
\hline Apoptosis & $\begin{array}{l}\text { Plasma membrane blebbing; reduction of } \\
\text { cellular and nuclear volume; nuclear } \\
\text { fragmentation; and chromatin } \\
\text { condensation }\end{array}$ & $\begin{array}{l}\text { Activation of caspases and } \\
\text { proapoptotic } \mathrm{Bcl}-2 \text { family } \\
\text { proteins, oligonucleosomal DNA } \\
\text { fragmentation, exposure of Plasma } \\
\text { membrane rupture, dissipation of } \\
\text { dissipation }\end{array}$ & $\begin{array}{l}\text { Positive: pro-apoptotic Bcl-2 } \\
\text { family proteins (Bax, Bak), p53; } \\
\text { Negative: anti-apoptotic Bcl-2 } \\
\text { family proteins (Bcl-2, Bcl-XL) }\end{array}$ \\
\hline Autophagy & $\begin{array}{l}\text { (Double-membraned) autolysosome } \\
\text { accumulation, cytoplasmic vacuolization }\end{array}$ & $\begin{array}{l}\text { Conversion from LC3-I to LC3-II, } \\
\text { degradation of p62Lck, Beclin-1 } \\
\text { dissociation from } \mathrm{Bcl}-2 / \mathrm{XL}\end{array}$ & $\begin{array}{l}\text { Positive: Beclin 1, ATG family } \\
\text { proteins (ATG5, ATG7) }\end{array}$ \\
\hline
\end{tabular}

drugs targeting apoptosis appear to be challenged with the occurrence of drug resistance and immune evasion in cancer treatment $(4,5)$.

Ferroptosis was firstly described as an iron-dependent form of non-apoptotic RCD induced by erastin in 2012, featured with excess reactive oxygen species (ROS) generation and lipid peroxidation (2). Unlike apoptosis and autophagy, ferroptosis is iron-dependent, with specific characteristics of cytological changes such as the rupture of outer mitochondrial membrane, small mitochondria with the condensed mitochondrial membrane, and a vanishing or decrease of mitochondria cristae (6-8). Based on the original studies of cell death, ferroptosis is markedly different from the other RCD types such as apoptosis and autophagy at levels of cell morphology, biochemical features, and regulations (Table 1) (9-11). Ferroptosis induction is associated with multiple disease occurrence, including immune system (nonalcoholic steatohepatitis), brain (stroke and intracerebral hemorrhage), neurodegenerative [Alzheimer's disease (AD), Huntington's disease (HD), and Parkinson's disease (PD)], heart (heart failure), and blood diseases (leukemia) $(7,12)$. Inhibiting ferroptosis has been identified as a potential prevention or therapeutic strategies for some of these diseases.

Beyond these findings, ferroptosis has recently gained much importance in cancer treatment, and emerging evidence shows that ferroptosis influences a growing number of oncogenic pathways. For example, P53 regulation promotes tumor cell ferroptosis but also decreases tumor metastases to blood, lung, and liver. Viswanathan and colleagues contended the contribution of ferroptosis to drug resistant phenomenon and cancer immunotherapeutic efficacy (13-15). Therefore, targeting ferroptosis might become a prospective strategy for cancer therapy, and its role in cancer-associated immune environment is worth deep exploration. In this review, we focus on the basic metabolisms and regulation of ferroptosis, its characteristics in cancer, and current research progress of ferroptosis-targeted therapies.

\section{Basis of ferroptosis}

The complicated interplay of iron, cysteine and lipid metabolism takes an important role in ferroptosis (Figure 1).

The most prominent character of ferroptosis is ROS generation, mainly caused by iron metabolism disorders (7). The endosomal uptake of circulated iron $\left(\mathrm{Fe}^{3+}\right)$ is mediated by its binding to transferrin (TF) and transferrin receptor 1 (TFR1). Iron $\mathrm{Fe}^{3+}$ is deoxidized to iron $\mathrm{Fe}^{2+}$, under the catalysis of iron oxide reductase named six-transmembrane epithelial antigen of the prostate 3 (STEAP3), and ultimately release into labile iron pool (LIP), due to $\mathrm{Fe}^{2+}$ s characteristics of high solubility and transfer electron capability. Increasing formation of LIP may trigger the Fenton reaction [the process of ROS generation mediated by interaction between $\mathrm{Fe}^{2+}$ and hydrogen peroxide $\left(\mathrm{H}_{2} \mathrm{O}_{2}\right)$ ], which may result in iron poisonous (16). Compared with RAS un-mutated ferroptosis-insensitive cells, RAS-mutated ferroptosis-sensitive cells increased the expression of TFR 1 and decreased the expression of ferritin light chain (FTL) and ferritin heavy chain 1 (FTH1) in the iron-storage protein subunits. This suggests 


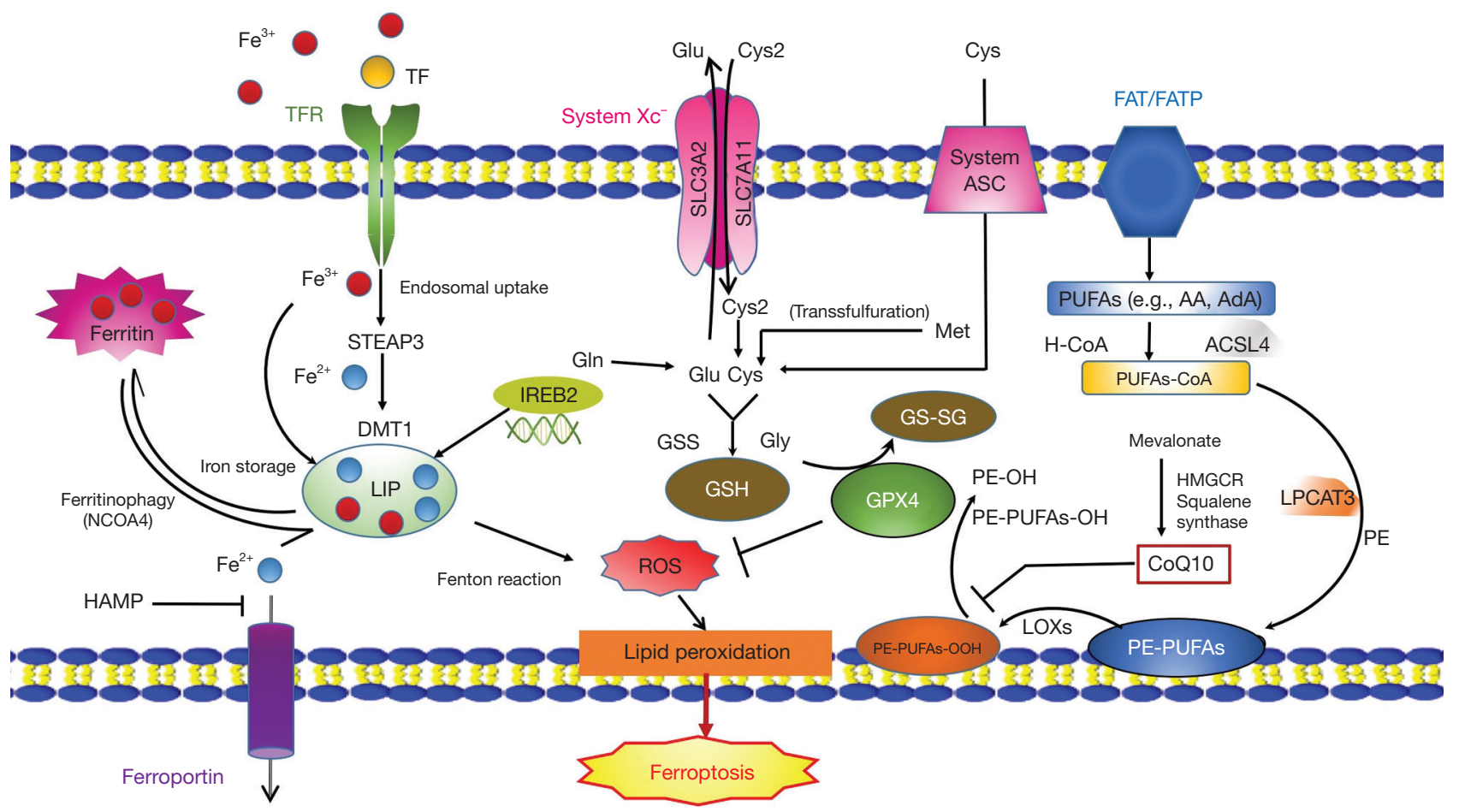

Figure 1 Mechanisms of ferroptosis. Mechanisms of Ferroptosis. Excess irons are regarded as an important factor for ferroptosis. The circulated iron $\left(\mathrm{Fe}^{3+}\right)$ combined with transferrin (TF) enters into cells mediated by transferrin receptor (TFR). Under the catalysis of iron oxide reductase STEAP3, $\mathrm{Fe}^{3+}$ can be deoxidized to $\mathrm{Fe}^{2+}$ and ultimately, releasing it into labile iron pool (LIP) mediated by DMT1. LIP consists of iron from endosomal uptake of circulated iron and ferritin degradation (ferritinophagy). System Xc-mediate the uptake of cystine (Cys2). Cys2, glutamate (Glu) and glycine (Gly) are materials of glutathione (GSH), which is an important antioxidant in cells. Transsulfurylation pathway may also increase the level of cysteine transformed from methionine (Met). Cysteine can be imported directly by alanine/serine/cysteine transporter (system ASC) under reducing conditions. The uptake of free PUAs such as arachidonic acid (AA) or adrenoxyl (AdA) mediated by fatty acid translocase (FAT) and fatty acid transport protein (FATP) can be converted to membrane phospholipids by enzyme acyl-CoA synthetase long-chain family member 4 (ACSL4) and lysophosphatidylcholine acyltransferase 3 (LPCAT3), which is important to ROS generation. PE-PUFAs can be oxidized to PE-PUFAs-OOH by lipoxygenases (LOXs), leading to ferroptosis. GPX4 roles as a protector to transfer PE-PUFAs-OOH to PE-OH. CoQ10, coenzyme Q10; DMT1, divalent metal transporter 1; FPN, ferroportin; Gln, glutamine; HAMP, hepcidin antimicrobial peptide; HMGCR, 3-hydroxy-3-methylglutaryl-CoA reductase; IREB2, iron-responsive element binding protein 2; NCOA4, Nuclear receptor coactivator 4; STEAP3: six-transmembrane epithelial antigen of the prostate 3.

that increasing iron intake and reducing iron storage may cause iron overload, in the end, leading to ferroptosis (17). Thus, intracellular iron metabolism homeostasis regulates ferroptosis process. Cancer cells become more vulnerable to iron toxicosis and ROS accumulation than noncancerous cells with a powerful iron dependency characteristic, making it possible for application of ferroptosis inducers in cancer therapy $(18,19)$.

System $\mathrm{Xc}^{-}$inhibition or enzyme glutathione peroxidase 4 (GPX4) inactivation can prompt ferroptosis as well. Antiporter system $\mathrm{Xc}^{-}$functioned to mediate the transmembrane exchange of extracellular cystine (Cys2) and intracellular glutamic acid (Glu) (20). The uptake of Cys2 is required for glutathione (GSH) formation, which is an important antioxidant interacting with GPX4 (21). The peroxidation of polyunsaturated fatty acids (PUFAs) is an important contributor for ferroptosis. Free PUFAs can be transformed into phosphatidylethanolamine (PE)-PUFAs$\mathrm{OOH}$ finally through three important enzymes including acyl-CoA synthetase long-chain family member 4 (ACSL4), 


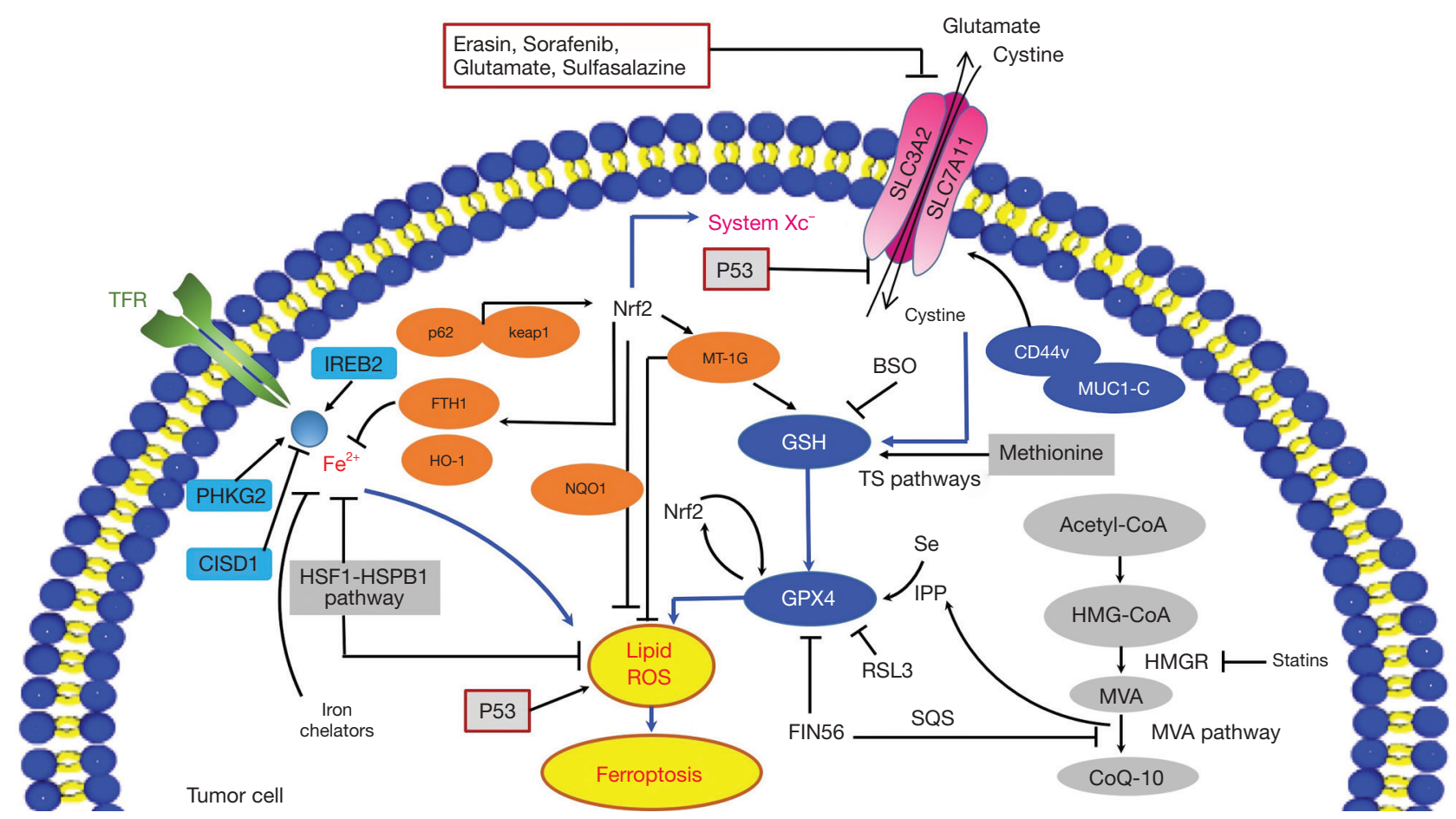

Figure 2 Ferroptosis modulation in tumor. Small molecules such as erastin, sorafenib, glutamate, and sulfasalazine induce ferroptosis by inhibiting system Xc- and impeding cysteine uptake, which could result in a subsequent decline of glutathione and a decrease of cells' antioxidative ability. mucin 1 C-terminal (MUC1-C) binds with CD44v to promote stability of the system Xc'. The cysteine level can also be supplemented by cellular methionine via the sulphur-transfer pathways. GPX4 can prevent ferroptosis by suppressing cellular lipid peroxides and the mevalonate (MVA) pathway is crucial for its maturation and the products of it (IPP and CoQ10) can promote synthesis of GPX4. Treatment FIN56 modulates squalene synthase (SQS) to reduce CoQ10. Ferroptosis inducer RSL3 can suppress GPX4 directly to regulate ferroptosis. The p62-Keap1-Nuclear factor (erythroid-derived 2)-like 2 (Nrf2) pathway is able to regulate Nrf2-targeted genes such as heme oxygenase-1 (HO-1), ferritin heavy chain 1 (FTH1), and NAD(P)H: quinone oxidoreductase 1 (NQO1) against ferroptosis. CISD1, PHKG2, and IREB2 are important in regulating iron metabolism and ferroptosis. Ironchelators can inhibit ferroptosis. The HSPB1 also impedes ferroptosis by inhibiting increase of intracellular iron. In addition, p53 also regulate ferroptosis through inhibiting SLC7A11 and promoting lipid peroxides production. BSO, buthionine sulfoximine; FTH1, ferritin heavy chain 1; HSP, heat-shock protein; HO-1, heme oxygenase-1; MUC1-C, mucin 1 C-terminal; MVA, mevalonate; NQO1, NAD(P)H: quinone oxidoreductase 1; Nrf2, nuclear factor (erythroid-derived 2)-like 2; SQS, squalene synthase.

lysophosphatidylcholine acyltransferase 3 (LPCAT3) and lipoxygenases (LOXs) (22).

Apart from the mechanisms discussed above, there are several other pathways involved in ferroptosis (Figure 2). In mevalonate (MVA) pathway, the activity of FIN56-targeted protein squalene synthase (SQS) reduces the idebenone level in cells, therefore decreasing the cellular antioxidation activity (23). Nuclear factor (erythroid-derived 2) like 2 ( Nrf2) is a regulator in the iron metabolism, and Nrf2 activation was reported capable of inhibiting ferroptosis in hepatocellular carcinoma cells (24). The famous tumor suppressor P53 also plays an essential role in ferroptosis regulation. Other pathways such as the sulphurtransfer pathway, heat shock factor-1 (HSF1)-heat shock protein beta-1 (HSPB1) pathway, and mucin $1 \mathrm{C}$-terminal (MUC1-C)/system $\mathrm{Xc}^{-}$(xCT) also contribute to ferroptosis regulation (Figure 2) (6). Further, evaluation of ferroptosisrelated molecules provides diverse approaches to monitor the ferroptosis process in-vitro and in-vivo (Tables 2,3).

\section{Hallmarks of ferroptosis in cancer}

\section{Role of ferroptosis in tumor suppression}

Tumor suppressor P53 inactivation is very common in 


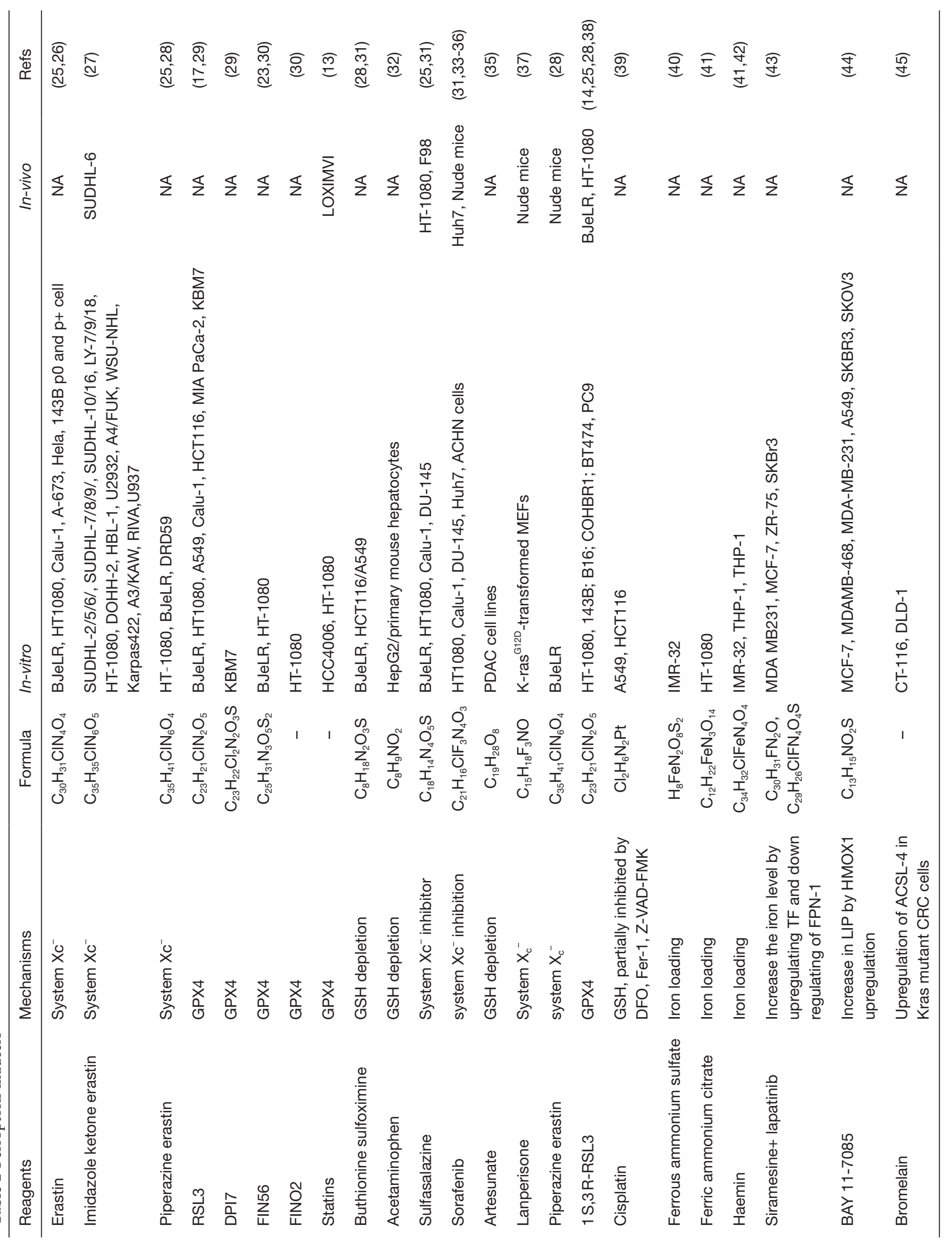




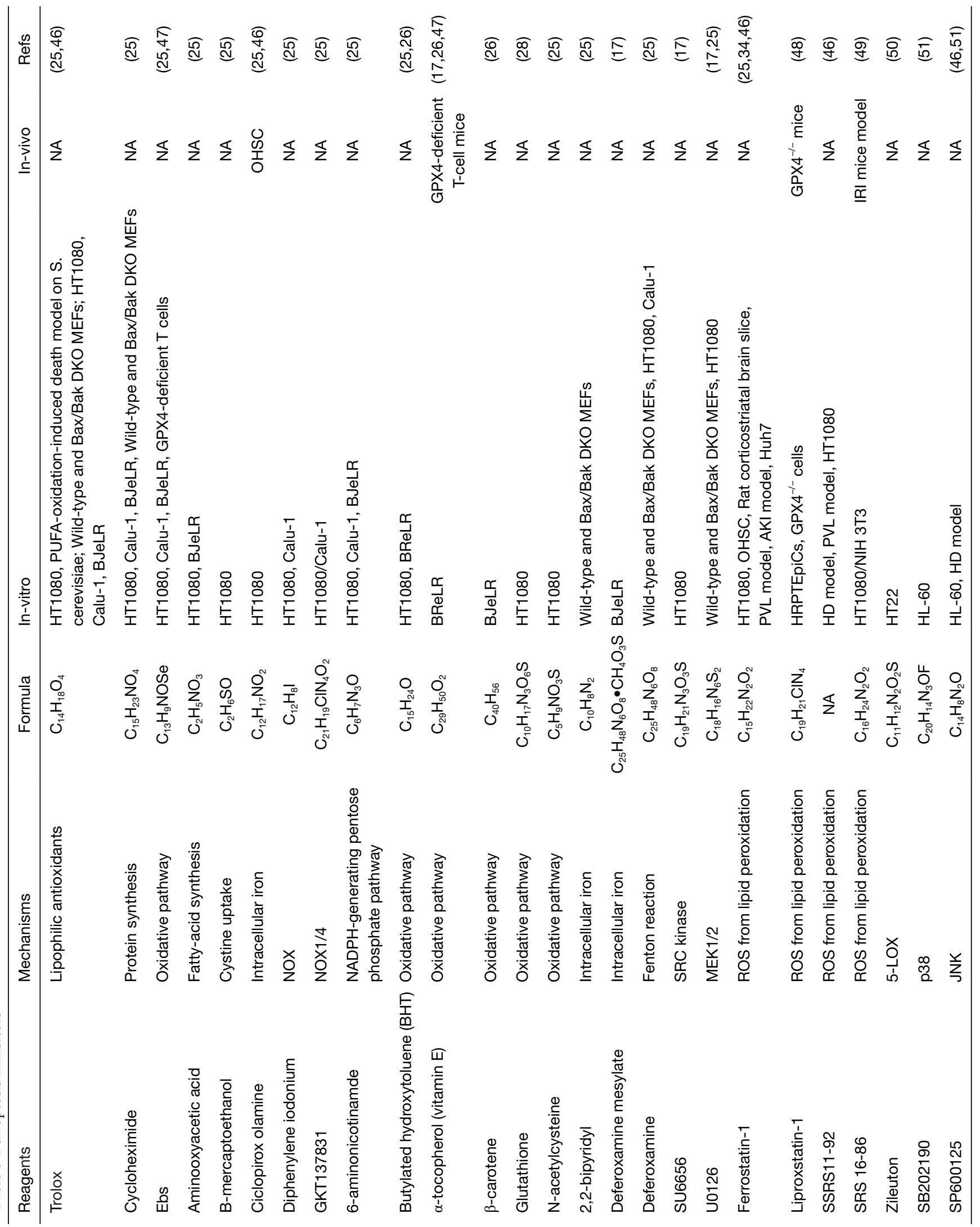




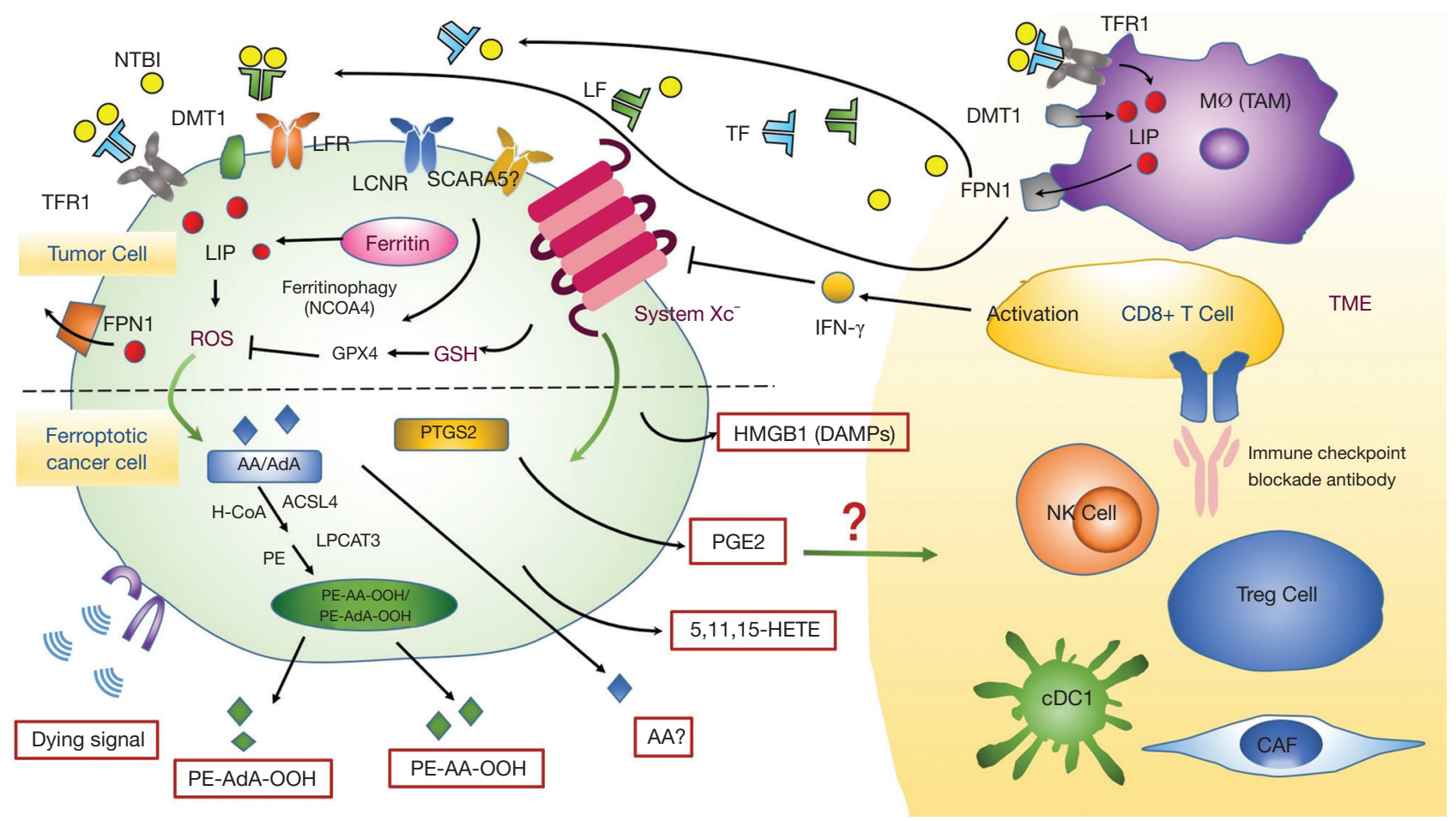

Figure 3 Role of ferroptosis in cancer. AA, arachidonic acid; AdA, adrenaline; ACSL4, acyl-CoA synthetase long-chain family 4; cDC1, type 1 dendritic cell; CAF, cancer-associated fibroblast; DMT1, divalent metal transporter 1; DAMP, damage-associated molecular pattern; HETE, hydroxyeicosatetraenoic acid; HMGB1, high mobility group box 1; FPN, ferroportin; LF, lactoferrin; LIP, labile iron pool; LCN, lipocalin; LPCAT3, lysophosphatidylcholine acyltransferase 3; NTBI, non-transferrin-bound iron; NCOA4, nuclear receptor coactivator 4; NK cell, natural killer cell; PE, phosphatidylethanolamine; PTGS2, prostaglandin-endoperoxide synthase 2; SCARA5, scavenger receptor A member 5; TF, transferrin; TFR1, transferrin receptor 1; TAM, tumor-associated macrophage; TME, tumor microenvironment.

cancers (52). The anti-tumor activity of P53 was thought to drive cell senescence, cell cycle arrest and apoptosis traditionally. These years, P53 has been explored to be essential in some other activities to suppress tumor progression $(53,54)$. In the study of Jiang's group, the acetylated defective mutant TP533KR lost its function to induce cell senescence, cell cycle arrest and apoptosis, while the function of ferroptosis-induction was still kept. The cancer progression was depressed through the inhibition of Cys2 uptake and elevation of tumors' sensitivity to ferroptosis by repressing the SLC7A11 expression (55). Additionally, evidence has shown a high release of mobility group box 1 (HMGB1) in ferroptosis. We may conjecture that ferroptotic tumor cells might be immunogenic (56). Release of damage-associated molecular patterns (DAMPs) can trigger Toll-like receptor 4 (TLR4) signals in ferroptotic cell death. Such phenomena have been observed in the attraction of neutrophils and dendritic cells (DCs), thus activating the innate immune system.

\section{Role of ferroptosis in tumor promotion and tumor evasion}

Ferroptotic cancer cells can release oxidized lipid mediators, which might regulate antitumor immunity (Figure 3). Eicosanoids such as 5-hydroxyeicosatetraenoic acid (5-HETE), 11-HETE and 15-HETE released from ferroptotic cells can induce GPX4 depletion and affect anti-tumor immunity (48). GPX4 inactivation was reported associated with ferroptosis promotion of $\mathrm{T}$ cells. The lower GPX4 activity is, the more pro-inflammatory lipid mediators such as 5-HETE and leukotriene B4 (LTB4) produced $(57,58)$, whereas LTB4, a kind of proinflammatory leukotriene, is crucial to carcinogenesis. Beyond free eicosanoids, esterified eicosanoids also role in immune response. Oxidized phosphatidylcholine was reported to inhibit DC maturation through Nrf2 activation and suppress the differentiation of T helper 17 (TH17) cells (59). Moreover, PUFA triacylglycerols and free PUFAs can cause inaccurate cross-presentation and defective 
antitumor immunity (60).

With further deeper studies, prostaglandins (PGs) have been regarded as significant immune regulators. Ferroptotic tumor cells were reported to associate with the increase of the release of prostaglandin E2 (PGE2), which acts as a main immunosuppressive factor and can affect anti-tumor immunity (Figure 3) (28). A study argued that PGE2 could downregulate chemokine receptors to block classical type 1 dendritic cells (cDC1s) directly as well as inhibit natural killer (NK) cells to secrete chemokines CC chemokine ligand 5 (CCL5) and chemokine lymphotactin (XCL1), so cDC1 accumulation was suppressed in tumor site. Additionally, PGE2 also functions in acquired immune system. Cytotoxic $\mathrm{T}$ cell action can be inhibited directly by PGE2 (61). Kurtova's study demonstrated a new mechanism of tumor cells' resistance to cytotoxic chemotherapy. They found under the treatment of chemotherapy, the PGE2 was released, which contributed to the repopulation of tumor cells (62).

\section{Association of ferroptosis with players (macrophages and lymphocytes) in tumor microenvironment (TME)}

Iron metabolism impacts the TME greatly and tumor cells contain more iron than normal cells (63). Malignant cells usually uptake iron mediated by TFR1. TFR1 overexpression has been observed in diverse cancers. The increase of labile iron in cancer cells is a double-edged sword, because it can facilitate DNA replication to accelerate tumor progression (64), but can also lead to ferroptosis (25).

The number and/or the distribution of tumor-associated macrophages (TAMs) may link to prognosis in potential malignant diseases (65). In most tumors, M2-polarized TAMs act as "iron-donators" that may promote cancer progression (66). Ferroportin (FPN-1) is a well-known cellular iron exporter, which can be regulated by hepcidin antimicrobial peptide (HAMP) (67). The cellular iron of M2-polarized TAMs is decreased in TME and the expression of FPN-1 is increased, thereby providing iron for tumor cells (68). However, an opposite viewpoint of TAM's role in cancer immune system was presented in a murine lung carcinoma model. This group demonstrated the hemolysis in the TME transformed the M2-polarized TAMs into M1-polarized TAMs, which functioned in inducing anti-cancer activity (69). Therefore, TAMs can both promote and suppress tumor progression. The association between lymphocytes and ferroptosis has also been studied. An elegant study from Wang's group in the ID8 ovarian tumor bearing mice model demonstrated the treatment of programmed death-ligand 1 (PD-L1) inhibitors can increase lipid ROS in CD45-IDB cells and suppress tumor progression. Their further study in erastin-resistant ID8 cells showed cells' insensitivity to anti-PD-L1 inhibitors, suggesting ferroptosis was involved in antitumor activity of immunotherapy. Besides, the cytokine interferon gamma (IFN- $\gamma$ ) released by CD8+ T cells could down-regulate the system $\mathrm{Xc}^{-}$expression in ferroptosis (Figure 3) (14).

\section{Hepcidin-ferroportin in ferroptosis of cancers}

Hepcidin and FPN1 constitute the vital regulator of the systemic iron homeostasis (63). As the negative regulator of protein FPN-1, hepcidin inhibits the release of iron from macrophages and intestinal mucosal cells into circulation. Systemic hepcidin is increased in patients with cancers such as prostate cancer (70), upper gastrointestinal tract tumors (71), breast cancer (72), and non-Hodgkin's lymphoma (73). Elevated plasma hepcidin could cause iron accumulation in cancer cells through degrading FPN1 , which would promote tumor progression by activating NF- $\kappa \mathrm{B}$ and Wnt signal pathways $(74,75)$. Besides, FPN1 knockdown in neuroblastoma cells could accelerate erastin-induced ferroptosis with increased iron-dependent ROS accumulation. Pancreatic cancer cells treated with ruscogenin were also observed elevated intracellular iron, ROS generation, and ferroptosis by downregulating FPN1 and upregulating TF (76). Thus, regulator hepcidinferroportin plays an important role in iron homeostasis and ferroptosis in cancers.

\section{MicroRNAs (miRNAs) in ferroptosis of cancers}

MiRNAs are a class of small non-coding RNA molecules, which can regulate the posttranscriptional gene expression. The important role of miRNAs in regulating iron homeostasis has been reviewed well before (77), including regulating proteins that control the iron export, import and storage. Based on this, emerging evidence suggests associations between different miRNAs and ferroptosis. For example, miR-137 was reported to regulate ferroptosis negatively in melanoma cells by regulating glutamine transporter SLC1A5 directly (78). Besides, cancerassociated fibroblasts could inhibit ferroptosis in tumor cells by secreting the exosomal miR-522, in which the lipid-ROS accumulation was blocked (79). The miR-4715-3p (80), miR-103a-3p (81), and miR-6852 (82) were also identified 
as regulators in ferroptosis in upper gastrointestinal, gastric, and lung cancers respectively. Thus, miRNAs play important roles in iron metabolisms and ferroptosis.

\section{Therapeutic strategies targeting ferroptosis}

\section{Small molecules to induce ferroptosis in cancer}

System $\mathrm{Xc}^{-}$determines the survival and growth of a large part of tumor cells to a certain extent, making it a possible target for cancer treatment.

Erastin is famous for its function directly inhibiting system $\mathrm{Xc}^{-}$to reduce the GSH level and induce ferroptosis. An interesting phenomenon was observed in Dixon's study in 2012 that erastin triggered the ROS accumulation in NRAS-mutant HT-1080 fibrosarcoma cells. Cell death was suppressed when adding iron chelator deferoxamine, which suggested erastin could induce ferroptosis (25). Further study has proved the importance of RAF/MEK/ERK signaling pathway in erastin-triggered ferroptosis in RASmutated cancer (7). Erastin derivatives such as piperazine erastin and imidazole ketone erastin (IKE) were developed to overcome the shortcomings of erastin such as poor water-solubility and unstable metabolism in-vivo. A study in the SUDHL6 xenograft animal model showed IKE was successfully used in the treatment of diffuse large B cell lymphoma (DLBCL) (27).

Another ferroptosis inducer is sorafenib, which is a multikinase inhibitor in therapy of cancers including advanced renal cell carcinoma, thyroid carcinoma and hepatocellular carcinoma. The cytotoxicity of sorafenib to hepatocellular carcinoma was removed when treated with iron chelator (33). However, resistance to sorafenib has emerged in some cancer cell lines. For example, sorafenib-induced ferroptosis was inhibited in hepatocellular carcinoma cells with retinoblastoma $(\mathrm{Rb})$ protein (34). The anti-inflammatory drug sulfasalazine (SAS) (brand name Salazopyrin, Sulazine, Azulfidine, etc.) can role as a ferroptosis inducer as well. In glioma cells, sulfasalazine can lead to ferroptosis by inhibiting system $\mathrm{Xc}^{-}$(83).

Some cancer cells induce ferroptosis by the transsulfuration pathway instead of system $\mathrm{Xc}^{-}$, and GPX4 inactivation can eradicate these tumor cells. One example is $(1 \mathrm{~S}, 3 \mathrm{R})-\mathrm{RSL}$, which induces ferroptosis by direct GPX4 inhibition. Another is FIN56, a specific inducer for ferroptosis as a GPX4 degradation promotor (23).

Beyond inhibition of system $\mathrm{Xc}^{-}$and GPX4, ferroptosis can also be induced by increasing LIP. For example, molecular BAY 11-7085 could induce ferroptosis through the Nrf2-SLC7A11-HO-1 pathway, and overexpression of heme oxygenase-1 (HO-1) that is encoded by HMOX1 has been observed in MDA-MB-231 breast cancer cells and DBTRG-05MG glioblastoma cells (44). Increased TF expression and decreased FPN-1 expression could also mediate ferroptosis using siramesine and lapatinib (43). In addition, autophagy also contributes to inducing ferroptosis by degrading ferritin in cancer cells. The cargo receptor nuclear receptor coactivator 4 (NCOA4) is important in the autophagic turnover of the ferritin in ferroptosis. In pancreatic cancer cells, overexpression of NCOA4 via gene transfection inhibited FIH1 expression and promoted erastin-induced ferroptosis (84). Further studies are needed to explore novel molecules targeting NCOA4 in ferroptosis to treat cancer. Beyond these small molecules, more are involved in ferroptosis induction (Table 2).

\section{Nanoparticle inducers of ferroptosis in cancer}

Nanotechnology applications have attracted much attention with specific physicochemical properties recently (Table 4).

Most nanomaterials such as iron-containing nanoparticles are based on Fenton reaction. Chen and colleagues developed a tumor-targeted nanoparticle named $\alpha$-enolase targeting peptide modified Pt-prodrug loaded $\mathrm{Fe}_{3} \mathrm{O}_{4}$ nanoparticles (ETP-PtFeNP). Tumor cells treated with ETP-PtFeNP were observed increased ROS generation, enhanced immunogenicity and strong anti-tumor immune response (99). Additionally, a novel nanoparticle called SRF@FeIIITA (SFT) was reported important in inhibiting tumor progression. By loading methylene blue $(\mathrm{MB})$ into SFT through depositing tannic acid (TA) and $\mathrm{Fe}^{3+}$ onto SRF nanocrystal, the combination therapy of photodynamic therapy (PDT) and ferroptosis succeeded (95).

Nanomaterials can also induce ferroptosis through GSH metabolism. The arginine-capped manganese silicate nanobubbles (AMSNs) were developed with a high efficiency of GSH depletion, based on the high ratio of surface area to volume (96). Further in-vivo study indicated AMSNs could help suppress Huh7 xenograft tumor growth by downregulating GPX4. This could be inhibited by ferroptosis inhibitor liproxstatin-1.

\section{Ferroptosis modulation for tumor sensitization to anticancer therapies}

Drug resistance becomes a major challenge in 


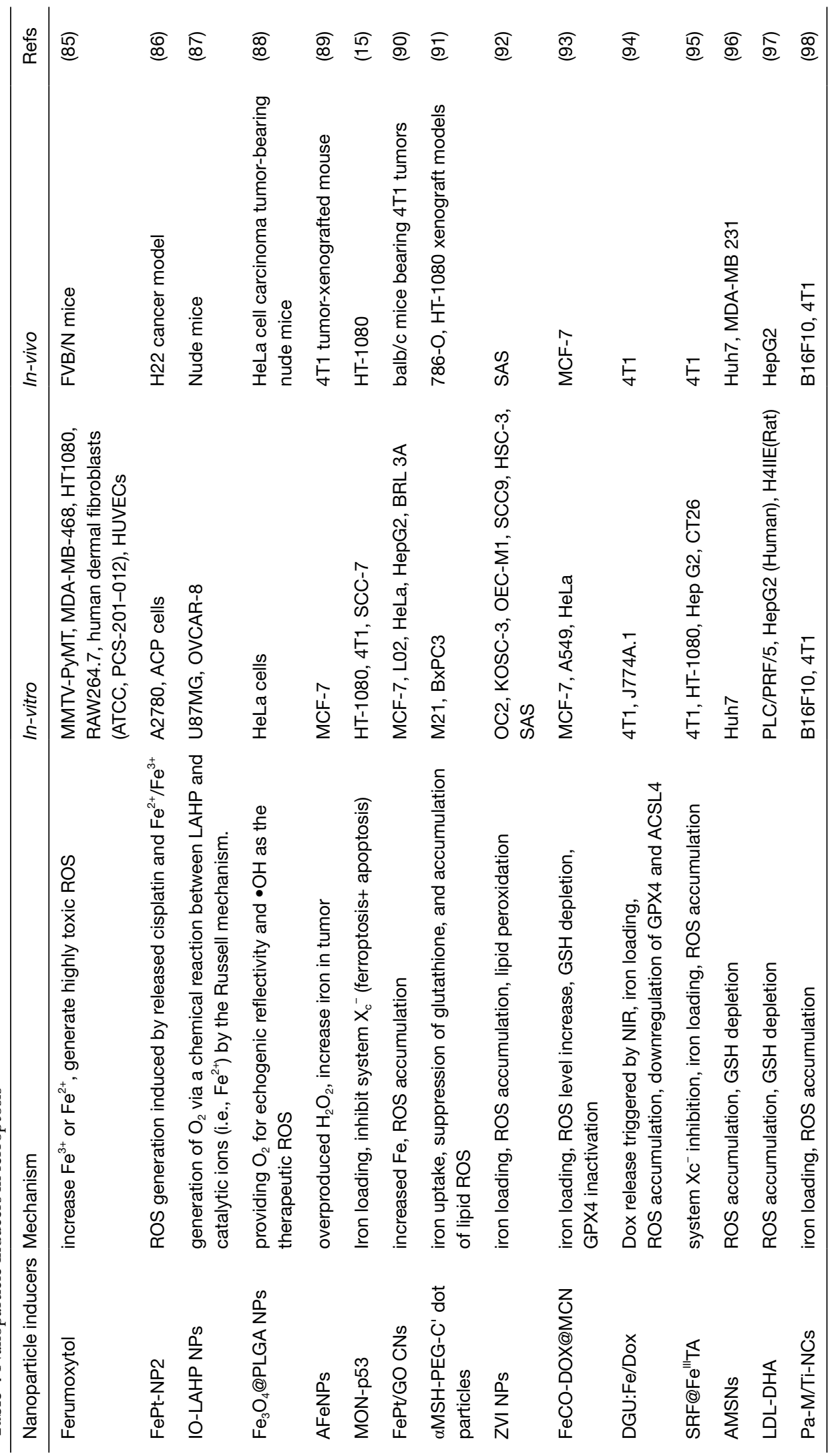


chemotherapy treatment. Hopefully, ferroptosis inducers may help to overcome this drug resistance (24), and combining ferroptosis agonists with chemotherapy drugs may become a novel strategy to cancer treatment. Persister cells refer to the cancer cells that remain its life after several rounds of chemotherapy drugs (100). Down-regulated Nrf2-targeted genes were shown in these cancer cells, while inhibition of intracellular NF2 and hippo signaling pathway can accelerate ferroptosis (101). Besides, decreased levels of GSH and nicotinamide adenine dinucleotide phosphate (NADPH) are observed in persister cells. Persister cells tend to be more sensitive to lipid peroxidation, and GPX4 inhibitors are proved lethal in persister cells. Thus, the ferroptosis inducers may be promising to overcome these cells' drug resistance.

\section{Ferroptosis-associated antitumor combination therapy}

Few studies have reported the combination of ferroptosis inducers with other anti-tumor therapies for clinical treatment. Many studies remain in the experimental stage. Iron-sulfur cluster biosynthetic enzyme NFS1 is important to cancer cells when the oxygen concentration is above $3-8 \%$ in most tissues. And high expression of it is often found in well-differentiated adenocarcinomas. The study of Alvarez SW's group suggested that inhibition of NFS1 cooperated with suppression of cysteine transport to induce tumor cell ferroptosis (102). Although some tumors are resistant to certain chemotherapy drugs, they are very sensitive to ferroptosis inducers. For example, pancreatic cancer cells are shown to be resistant to chemotherapyinduced apoptosis, but have great sensitivity to artemisinininduced ferroptosis (103). Therefore, ferroptosis inducers turn out to be a promising strategy for cancer therapies.

\section{Targeting ferroptosis: potential way to prevent tumor metastasis}

Clinical treatment of tumor metastasis seems complicated possibly because of tumor heterogeneity, activity of oncogenes, epithelial-mesenchymal transition (EMT), and the microenvironment of the metastatic sites (104). Cancer metastasis can be inhibited by high intracellular oxidative stress. Hence, targeting ferroptosis may become a promising way to prevent metastasis.

Nanoparticles may offer enormous advantages to treat cancer metastasis because of their relatively low risk compared with locally injected agents (105). Based on coordination between ferric iron $\left(\mathrm{Fe}^{3+}\right)$ and tannic acid (TA), p53 plasmid-encapsulated metal-organic network (MON-p53) was developed. Treatment of MON-p53 could suppress cancer cell migration in a wound healing assay in-vitro (15), indicating that MON-p53 might inhibit tumor metastasis. Mesenchymal cancer cells are easily metastatic and prone to be resistant to anticancer treatments (13). Increased cell sensitivity to chemotherapy drugs can also reduce cancer metastasis to some extent. Antagonizing NF2-YAP pathway allows the promotion of ferroptosis by up-regulating ferroptosis modulators such as ACSL4 and transferrin receptor (TFRC) (101). This provides a novel insight that mesenchymal or metastatic property cancer cells are highly sensitive to ferroptosis.

\section{Conclusions}

Ferroptosis is a new form of RCD, characterized by lethal ROS accumulation and over production of lipid peroxidation, which relates closely to excess iron loading, GSH depletion as well as lipid peroxidation. An important peroxidase GPX4 can protect cells from ferroptosis and inactivation of GPX4 will lead to ROS accumulation. Ferroptosis can also be regulated by pathways including mevalonate pathway, P53 pathway and Nrf2. Researchers have increasingly explored the role of ferroptosis in TME. Tumor cells are proved to contain more intracellular iron than normal cells, related to the over-expression of TFR on tumor cells and iron supply of macrophages. Ferroptosis roles as a double-edged sword in tumor development because ferroptotic cancer cells release a variety of signaling molecules either to inhibit tumor growth or to promote tumor proliferation. The role of these signals released from ferroptotic cancer cells in TME remains further investigation.

Appropriate drug type and dose of ferroptosis inducers have a certain therapeutic effect on different types of tumors, making ferroptosis inducers prospective to treat cancer. A vast majority of studies on ferroptosis inducers are still in the experimental phase. Interestingly, ferroptosis is applicable to those tumor cells that are less sensitive to chemotherapy, radiotherapy, and other treatments.

However, some considerations should be taken into account for treating cancers based on ferroptosis. Ferroptosis is identified related to various pathological cell deaths and the occurrence of many diseases. Degenerative pathological changes may occur due to the reduced ability to repair lipid peroxidation (106). Therefore, 
ferroptosis is extremely complex in human health and diseases. Ferroptosis inducers can not only treat cancer, but also promote cancer and other diseases. The specificity and optimal dose of ferroptosis inducers need further exploration, so damages to normal cells can be reduced. In addition, the heterogeneity and replasticity of tumor cells affect their sensitivity to ferroptosis inducers differently, and the specific functions of signals released from ferroptotic cancer cells in TME has not been determined.

With deeper understanding of ferroptosis and its relationship with cancers, the mystery of ferroptosis will be gradually unveiled in the near future.

\section{Acknowledgments}

Funding: This study was supported in part by two grant from National Natural Science Foundation of China (81871865, 81972169), Shanghai Science and Technology Commission (19411950301), Shanghai Health and Construction Commission: Shanghai Clinical Key Specialty Construction Project, Shanghai Shenkang Hospital Development Center: Shanghai Major Diseases Multidisciplinary Cooperative Diagnosis and Treatment Capacity Building Project, and 2014 Shanghai Leading Talent Project.

\section{Footnote}

Peer Review File: Available at http://dx.doi.org/10.21037/ tlcr-20-341

Conflicts of Interest: All authors have completed the ICMJE uniform disclosure form (available at http://dx.doi. org/10.21037/tlcr-20-341). CZ serves as an unpaid editorial board member of Translational Lung Cancer Research from Mar 2012 to Mar 2022. The other authors have no conflicts of interest to declare.

Ethical Statement: The authors are accountable for all aspects of the work in ensuring that questions related to the accuracy or integrity of any part of the work are appropriately investigated and resolved.

Open Access Statement: This is an Open Access article distributed in accordance with the Creative Commons Attribution-NonCommercial-NoDerivs 4.0 International License (CC BY-NC-ND 4.0), which permits the noncommercial replication and distribution of the article with the strict proviso that no changes or edits are made and the original work is properly cited (including links to both the formal publication through the relevant DOI and the license). See: https://creativecommons.org/licenses/by-nc-nd/4.0/.

\section{References}

1. Fricker M, Tolkovsky AM, Borutaite V, et al. Neuronal Cell Death. Physiol Rev 2018;98:813-80.

2. Tang D, Kang R, Berghe TV, et al. The molecular machinery of regulated cell death. Cell Res 2019;29:347-64.

3. Gao M, Jiang X. To eat or not to eat-the metabolic flavor of ferroptosis. Curr Opin Cell Biol 2018;51:58-64.

4. Janson V, Johansson A, Grankvist K. Resistance to caspase- 8 and -9 fragments in a malignant pleural mesothelioma cell line with acquired cisplatin-resistance. Cell Death Dis 2010;1:e78.

5. Han J, Lv W, Sheng H, et al. Ecliptasaponin A induces apoptosis through the activation of ASK1/JNK pathway and autophagy in human lung cancer cells. Ann Transl Med 2019;7:539.

6. Cao JY, Dixon SJ. Mechanisms of ferroptosis. Cell Mol Life Sci 2016;73:2195-209.

7. Xie $Y$, Hou W, Song $X$, et al. Ferroptosis: process and function. Cell Death Differ 2016;23:369-79.

8. Yu H, Guo P, Xie X, et al. Ferroptosis, a new form of cell death, and its relationships with tumourous diseases. J Cell Mol Med 2017;21:648-57.

9. Galluzzi L, Vitale I, Abrams JM, et al. Molecular definitions of cell death subroutines: recommendations of the Nomenclature Committee on Cell Death 2012. Cell Death Differ 2012;19:107-20.

10. Kroemer G, El-Deiry WS, Golstein P, et al. Classification of cell death: recommendations of the Nomenclature Committee on Cell Death. Cell Death Differ 2005;12 Suppl 2:1463-7.

11. Kroemer G, Galluzzi L, Vandenabeele P, et al. Classification of cell death: recommendations of the Nomenclature Committee on Cell Death 2009. Cell Death Differ 2009;16:3-11.

12. Qiu Y, Cao Y, Cao W, et al. The Application of Ferroptosis in Diseases. Pharmacol Res 2020;159:104919.

13. Viswanathan VS, Ryan MJ, Dhruv HD, et al. Dependency of a therapy-resistant state of cancer cells on a lipid peroxidase pathway. Nature 2017;547:453.

14. Wang W, Green M, Choi JE, et al. CD8(+) T cells regulate tumour ferroptosis during cancer immunotherapy. Nature 2019;569:270-4.

15. Zheng DW, Lei Q, Zhu JY, et al. Switching Apoptosis to 
Ferroptosis: Metal-Organic Network for High-Efficiency Anticancer Therapy. Nano Lett 2017;17:284-91.

16. Lachaier E, Louandre C, Ezzoukhry Z, et al. Ferroptosis, a new form of cell death relevant to the medical treatment of cancer. Med Sci (Paris) 2014;30:779-83.

17. Yang WS, Stockwell BR. Synthetic lethal screening identifies compounds activating iron-dependent, nonapoptotic cell death in oncogenic-RAS-harboring cancer cells. Chem Biol 2008;15:234-45.

18. Hassannia B, Vandenabeele P, Vanden Berghe T. Targeting Ferroptosis to Iron Out Cancer. Cancer Cell 2019;35:830-49.

19. Lei P, Bai T, Sun YL. Mechanisms of Ferroptosis and Relations With Regulated Cell Death: A Review. Front Physiol 2019;10:139.

20. Liang C, Zhang X, Yang M, et al. Recent Progress in Ferroptosis Inducers for Cancer Therapy. Adv Mater 2019;31:e1904197.

21. Eagle H. Nutrition needs of mammalian cells in tissue culture. Science 1955;122:501-14.

22. Kagan VE, Mao G, Qu F, et al. Oxidized arachidonic and adrenic PEs navigate cells to ferroptosis. Nat Chem Biol 2017;13:81-90.

23. Shimada K, Skouta R, Kaplan A, et al. Global survey of cell death mechanisms reveals metabolic regulation of ferroptosis. Nat Chem Biol 2016;12:497-503.

24. Shin D, Kim EH, Lee J, et al. Nrf2 inhibition reverses resistance to GPX4 inhibitor-induced ferroptosis in head and neck cancer. Free Radic Biol Med 2018;129:454-62.

25. Dixon SJ, Lemberg KM, Lamprecht MR, et al. Ferroptosis: an iron-dependent form of nonapoptotic cell death. Cell 2012;149:1060-72.

26. Yagoda N, von Rechenberg M, Zaganjor E, et al. RAS-RAF-MEK-dependent oxidative cell death involving voltage-dependent anion channels. Nature 2007;447:864-8.

27. Zhang Y, Tan H, Daniels JD, et al. Imidazole Ketone Erastin Induces Ferroptosis and Slows Tumor Growth in a Mouse Lymphoma Model. Cell Chem Biol 2019;26:623-33.e9.

28. Yang WS, SriRamaratnam R, Welsch ME, et al. Regulation of ferroptotic cancer cell death by GPX4. Cell 2014;156:317-31.

29. Dixon SJ, Winter GE, Musavi LS, et al. Human Haploid Cell Genetics Reveals Roles for Lipid Metabolism Genes in Nonapoptotic Cell Death. ACS Chem Biol 2015;10:1604-9.

30. Gaschler MM, Andia AA, Liu HR, et al. FINO2 initiates ferroptosis through GPX4 inactivation and iron oxidation. Nat Chem Biol 2018;14:507.

31. Dixon SJ, Patel DN, Welsch M, et al. Pharmacological inhibition of cystine-glutamate exchange induces endoplasmic reticulum stress and ferroptosis. Elife. 2014;3:e02523.

32. Lőrincz T, Jemnitz K, Kardon T, et al. Ferroptosis is Involved in Acetaminophen Induced Cell Death. Pathol Oncol Res 2015;21:1115-21.

33. Hou W, Xia H, Zhou S, et al. The MEK inhibitors enhance the efficacy of sorafenib against hepatocellular carcinoma cells through reducing p-ERK rebound. Transl Cancer Res 2019;8:1224-32.

34. Louandre C, Marcq I, Bouhlal H, et al. The retinoblastoma $(\mathrm{Rb})$ protein regulates ferroptosis induced by sorafenib in human hepatocellular carcinoma cells. Cancer Lett 2015;356:971-7.

35. Eling N, Reuter L, Hazin J, et al. Identification of artesunate as a specific activator of ferroptosis in pancreatic cancer cells. Oncoscience 2015;2:517-32.

36. Louandre C, Ezzoukhry Z, Godin C, et al. Iron-dependent cell death of hepatocellular carcinoma cells exposed to sorafenib. Int J Cancer 2013;133:1732-42.

37. Shaw AT, Winslow MM, Magendantz M, et al. Selective killing of K-ras mutant cancer cells by small molecule inducers of oxidative stress. Proc Natl Acad Sci U S A 2011;108:8773-8.

38. Hangauer MJ, Viswanathan VS, Ryan MJ, et al. Drugtolerant persister cancer cells are vulnerable to GPX4 inhibition. Nature 2017;551:247-50.

39. Guo J, Xu B, Han Q, et al. Ferroptosis: A Novel Anti-tumor Action for Cisplatin. Cancer Res Treat 2018;50:445-60.

40. Hassannia B, Wiernicki B, Ingold I, et al. Nano-targeted induction of dual ferroptotic mechanisms eradicates highrisk neuroblastoma. J Clin Invest 2018;128:3341-55.

41. Fang S, Yu X, Ding H, et al. Effects of intracellular iron overload on cell death and identification of potent cell death inhibitors. Biochem Biophys Res Commun 2018;503:297-303.

42. Imoto S, Kono M, Suzuki T, et al. Haemin-induced cell death in human monocytic cells is consistent with ferroptosis. Transfus Apher Sci 2018;57:524-31.

43. Ma S, Henson EE, Chen Y, et al. Ferroptosis is induced following siramesine and lapatinib treatment of breast cancer cells. Cell Death Dis 2016;7:e2307.

44. Chang LC, Chiang SK, Chen SE, et al. Heme oxygenase-1 mediates BAY 11-7085 induced ferroptosis. Cancer Lett 
2018;416:124-37.

45. Park S, Oh J, Kim M, et al. Bromelain effectively suppresses Kras-mutant colorectal cancer by stimulating ferroptosis. Anim Cells Syst (Seoul) 2018;22:334-40.

46. Skouta R, Dixon SJ, Wang J, et al. Ferrostatins inhibit oxidative lipid damage and cell death in diverse disease models. J Am Chem Soc 2014;136:4551-6.

47. Iannetti A, Pacifico F, Acquaviva R, et al. The neutrophil gelatinase-associated lipocalin (NGAL), a NF-kappaBregulated gene, is a survival factor for thyroid neoplastic cells. Proc Natl Acad Sci U S A 2008;105:14058-63.

48. Friedmann Angeli JP, Schneider M, Proneth B, et al. Inactivation of the ferroptosis regulator $\mathrm{Gpx} 4$ triggers acute renal failure in mice. Nat Cell Biol 2014;16:1180-91.

49. Linkermann A, Skouta R, Himmerkus N, et al. Synchronized renal tubular cell death involves ferroptosis. Proc Natl Acad Sci U S A 2014;111:16836-41.

50. Liu Y, Wang W, Li Y, et al. The 5-Lipoxygenase Inhibitor Zileuton Confers Neuroprotection against Glutamate Oxidative Damage by Inhibiting Ferroptosis. Biol Pharm Bull 2015;38:1234-9.

51. Yu Y, Xie Y, Cao L, et al. The ferroptosis inducer erastin enhances sensitivity of acute myeloid leukemia cells to chemotherapeutic agents. Mol Cell Oncol 2015;2:e1054549.

52. Berkers CR, Maddocks ODK, Cheung EC, et al. Metabolic Regulation by p53 Family Members. Cell Metab 2013;18:617-33.

53. Valente LJ, Gray DHD, Michalak EM, et al. p53 Efficiently Suppresses Tumor Development in the Complete Absence of Its Cell-Cycle Inhibitory and Proapoptotic Effectors p21, Puma, and Noxa. Cell Rep 2013;3:1339-45.

54. Brady CA, Jiang D, Mello SS, et al. Distinct p53 transcriptional programs dictate acute DNA-damage responses and tumor suppression. Cell 2011;145:571-83.

55. Jiang L, Kon N, Li TY, et al. Ferroptosis as a p53mediated activity during tumour suppression. Nature 2015;520:57.

56. Friedmann Angeli JP, Krysko DV, Conrad M. Ferroptosis at the crossroads of cancer-acquired drug resistance and immune evasion. Nat Rev Cancer 2019;19:405-14.

57. Li C, Deng X, Zhang W, et al. Novel Allosteric Activators for Ferroptosis Regulator Glutathione Peroxidase 4. J Med Chem 2019;62:266-75.

58. Matsushita M, Freigang S, Schneider C, et al. T cell lipid peroxidation induces ferroptosis and prevents immunity to infection. J Exp Med 2015;212:555-68.
59. Rothe T, Gruber F, Uderhardt S, et al.

12/15-Lipoxygenase-mediated enzymatic lipid oxidation regulates DC maturation and function. J Clin Invest 2015;125:1944-54.

60. Ramakrishnan R, Tyurin VA, Veglia F, et al. Oxidized lipids block antigen cross-presentation by dendritic cells in cancer. J Immunol 2014;192:2920-31.

61. Wang D, DuBois R. Immunosuppression associated with chronic inflammation in the tumor microenvironment. Carcinogenesis 2015;36:1085-93.

62. Kurtova AV, Xiao J, Mo Q, et al. Blocking PGE2induced tumour repopulation abrogates bladder cancer chemoresistance. Nature 2015;517:209-13.

63. Pfeifhofer-Obermair C, Tymoszuk P, Petzer V, et al. Iron in the Tumor Microenvironment-Connecting the Dots. Front Oncol 2018;8:549.

64. Thanan R, Oikawa S, Yongvanit P, et al. Inflammationinduced protein carbonylation contributes to poor prognosis for cholangiocarcinoma. Free Radic Biol Med 2012;52:1465-72.

65. Bingle L, Brown NJ, Lewis CE. The role of tumourassociated macrophages in tumour progression: implications for new anticancer therapies. J Pathol 2002;196:254-65.

66. Cairo G, Recalcati S, Mantovani A, et al. Iron trafficking and metabolism in macrophages: contribution to the polarized phenotype. Trends Immunol 2011;32:241-7.

67. Nemeth E, Tuttle MS, Powelson J, et al. Hepcidin regulates cellular iron efflux by binding to ferroportin and inducing its internalization. Science 2004;306:2090-3.

68. Ganz T. Macrophages and Iron Metabolism. Microbiol Spectr 2016;4.

69. Costa da Silva M, Breckwoldt MO, Vinchi F, et al. Iron Induces Anti-tumor Activity in Tumor-Associated Macrophages. Front Immunol 2017;8:1479.

70. Tanno T, Rabel A, Alleyne M, et al. Hepcidin, anaemia, and prostate cancer. BJU Int 2011;107:678-9.

71. Macciò A, Madeddu C, Gramignano G, et al. The role of inflammation, iron, and nutritional status in cancer-related anemia: results of a large, prospective, observational study. Haematologica 2015;100:124-32.

72. Lamy PJ, Durigova A, Jacot W. Iron homeostasis and anemia markers in early breast cancer. Clin Chim Acta 2014;434:34-40.

73. Tisi MC, Bozzoli V, Giachelia M, et al. Anemia in diffuse large B-cell non-Hodgkin lymphoma: the role of interleukin-6, hepcidin and erythropoietin. Leuk Lymphoma 2014;5 5:270-5. 
74. Brookes MJ, Boult J, Roberts $\mathrm{K}$, et al. A role for iron in Wnt signalling. Oncogene 2008;27:966-75.

75. Xiong S, She H, Takeuchi H, et al. Signaling role of intracellular iron in NF-kappaB activation. J Biol Chem 2003;278:17646-54.

76. Song Z, Xiang X, Li J, et al. Ruscogenin induces ferroptosis in pancreatic cancer cells. Oncol Rep 2020;43:516-24.

77. Manz DH, Blanchette NL, Paul BT, et al. Iron and cancer: recent insights. Ann N Y Acad Sci 2016;1368:149-61.

78. Luo M, Wu L, Zhang K, et al. miR-137 regulates ferroptosis by targeting glutamine transporter SLC1A5 in melanoma. Cell Death Differ 2018;25:1457-72.

79. Zhang H, Deng T, Liu R, et al. CAF secreted miR-522 suppresses ferroptosis and promotes acquired chemoresistance in gastric cancer. Mol Cancer 2020;19:43.

80. Gomaa A, Peng D, Chen Z, et al. Epigenetic regulation of AURKA by miR-4715-3p in upper gastrointestinal cancers. Sci Rep 2019;9:16970.

81. Niu Y, Zhang J, Tong Y, et al. Physcion 8-O-betaglucopyranoside induced ferroptosis via regulating miR-103a-3p/GLS2 axis in gastric cancer. Life Sci 2019;237:116893.

82. Wang M, Mao C, Ouyang LL, et al. Long noncoding RNA LINC00336 inhibits ferroptosis in lung cancer by functioning as a competing endogenous RNA (vol 26, pg 2329, 2019). Cell Death Differ 2020;27:1447.

83. Sehm T, Fan Z, Ghoochani A, et al. Sulfasalazine impacts on ferroptotic cell death and alleviates the tumor microenvironment and glioma-induced brain edema. Oncotarget 2016;7:36021-33.

84. Hou W, Xie Y, Song X, et al. Autophagy promotes ferroptosis by degradation of ferritin. Autophagy 2016;12:1425-8.

85. Zanganeh S, Hutter G, Spitler R, et al. Iron oxide nanoparticles inhibit tumour growth by inducing proinflammatory macrophage polarization in tumour tissues. Nat Nanotechnol 2016;11:986-94.

86. Ma P, Xiao H, Yu C, et al. Enhanced Cisplatin Chemotherapy by Iron Oxide Nanocarrier-Mediated Generation of Highly Toxic Reactive Oxygen Species. Nano Lett 2017;17:928-37.

87. Zhou Z, Song J, Tian R, et al. Activatable Singlet Oxygen Generation from Lipid Hydroperoxide Nanoparticles for Cancer Therapy. Angew Chem Int Ed Engl 2017;56:6492-6.

88. Li WP, Su CH, Chang YC, et al. Ultrasound-Induced Reactive Oxygen Species Mediated Therapy and Imaging
Using a Fenton Reaction Activable Polymersome. Acs Nano 2016;10:2017-27.

89. Zhang C, Bu WB, Ni DL, et al. Synthesis of Iron Nanometallic Glasses and Their Application in Cancer Therapy by a Localized Fenton Reaction. Angew Chem Int Ed Engl 2016;55:2101-6.

90. Yue L, Wang J, Dai Z, et al. pH-Responsive, SelfSacrificial Nanotheranostic Agent for Potential In Vivo and In Vitro Dual Modal MRI/CT Imaging, Real-Time, and In Situ Monitoring of Cancer Therapy. Bioconjug Chem 2017;28:400-9.

91. Kim SE, Zhang L, Ma K, et al. Ultrasmall nanoparticles induce ferroptosis in nutrient-deprived cancer cells and suppress tumour growth. Nat Nanotechnol 2016;11:977-85.

92. Huang KJ, Wei YH, Chiu YC, et al. Assessment of zero-valent iron-based nanotherapeutics for ferroptosis induction and resensitization strategy in cancer cells. Biomater Sci 2019;7:1311-22.

93. Yao X, Yang P, Jin Z, et al. Multifunctional nanoplatform for photoacoustic imaging-guided combined therapy enhanced by $\mathrm{CO}$ induced ferroptosis. Biomaterials 2019;197:268-83.

94. Bao W, Liu X, Lv Y, et al. Nanolongan with Multiple OnDemand Conversions for Ferroptosis-Apoptosis Combined Anticancer Therapy. Acs Nano 2019;13:260-73.

95. Liu T, Liu W, Zhang M, et al. Ferrous-SupplyRegeneration Nanoengineering for Cancer-Cell-Specific Ferroptosis in Combination with Imaging-Guided Photodynamic Therapy. Acs Nano 2018;12:12181-92.

96. Wang S, Li FY, Qiao RR, et al. Arginine-Rich Manganese Silicate Nanobubbles as a FerroptosisInducing Agent for Tumor-Targeted Theranostics. Acs Nano 2018;12:12380-92.

97. Ou W, Mulik RS, Anwar A, et al. Low-density lipoprotein docosahexaenoic acid nanoparticles induce ferroptotic cell death in hepatocellular carcinoma. Free Radic Biol Med 2017;112:597-607.

98. Zhang F, Li F, Lu GH, et al. Engineering Magnetosomes for Ferroptosis/Immunomodulation Synergism in Cancer. Acs Nano 2019;13:5662-73.

99. Chen Q, Liu L, Lu Y, et al. Tumor MicroenvironmentTriggered Aggregated Magnetic Nanoparticles for Reinforced Image-Guided Immunogenic Chemotherapy. Adv Sci (Weinh) 2019;6:1802134.

100. Sharma SV, Lee DY, Li B, et al. A chromatin-mediated reversible drug-tolerant state in cancer cell subpopulations. Cell 2010;141:69-80.

101.Wu J, Minikes AM, Gao M, et al. Intercellular interaction 
dictates cancer cell ferroptosis via NF2-YAP signalling. Nature 2019;572:402-6.

102. Alvarez SW, Sviderskiy VO, Terzi EM, et al. NFS1 undergoes positive selection in lung tumours and protects cells from ferroptosis. Nature 2017;551:639-43.

103. Efferth T. From ancient herb to modern drug: Artemisia annua and artemisinin for cancer therapy. Semin Cancer Biol 2017;46:65-83.

104. Wan L, Pantel K, Kang YB. Tumor metastasis: moving new biological insights into the clinic. Nat Med 2013;19:1450-64.

105. Cabral H, Makino J, Matsumoto Y, et al. Systemic Targeting of Lymph Node Metastasis through the Blood Vascular System by Using Size-Controlled Nano carriers. Acs Nano 2015;9:4957-67.

106. Alim I, Caulfield JT, Chen Y, et al. Selenium Drives a Transcriptional Adaptive Program to Block Ferroptosis and Treat Stroke. Cell 2019;177:1262-79.e25.

Cite this article as: Jiang M, Qiao M, Zhao C, Deng J, Li X, Zhou C. Targeting ferroptosis for cancer therapy: exploring novel strategies from its mechanisms and role in cancers. Transl Lung Cancer Res 2020;9(4):1569-1584. doi: 10.21037/tlcr-20-341 